\title{
CT reconstruction from portal images acquired during volumetric modulated arc therapy
}

\author{
G Poludniowski, M D R Thomas, P M Evans and S Webb \\ Joint Department of Physics, Institute of Cancer Research and Royal Marsden NHS \\ Foundation Trust, Downs Road, Sutton, Surrey, SM2 5PT UK \\ E-mail: Gavin.Poludniowski@icr.ac.uk
}

\begin{abstract}
Volumetric Modulated Arc Therapy (VMAT), a form of Intensity Modulated Arc Therapy (IMAT), has become a topic of research and clinical activity in recent years. As a form of arc therapy, portal images acquired during the treatment fraction form a (partial) Radon transform of the patient. We show that these portal images, when used in a modified global cone-beam Filtered Back-Projection (FBP) algorithm, allow a surprisingly recognizable CT-volume to be reconstructed. The possibility of distinguishing anatomy in such VMAT-CT reconstructions, suggests that this could prove to be a valuable treatment position-verification tool. Further, some potential for local-tomography techniques to improve image quality is shown.
\end{abstract}

PACS: 87.57.-s, 87.57.Q-, 87.57.nf, 87.56.-v

\section{Introduction}

Intensity Modulated Arc Therapy (IMAT) is not a new concept (Yu 1995). However, the potential advantages of a particular form of IMAT, volumetric modulated arc therapy (VMAT), have recently given rise to a number of commercial implementations and research studies (Otto 2008, Palma et al 2008, Bedford et al 2008, Bedford 2009, Vebakel et al 2009, Bortfeld and Webb 2009). In these systems, the dose rate, gantry rotation speed and multi-leaf collimator (MLC) positions all vary during delivery. In general, plans comparable in quality and accuracy to static-gantry intensity-modulated radiotherapy (IMRT) can be obtained, normally with reduced delivery times (Bedford 2009, Verbakel et al 2009). We note that how and why VMAT shows such effectiveness is not yet well-understood (Webb and McQuaid 2009) and not all the potential equipment options are yet implemented and optimized (Webb 2010). A number of authors have discussed image-guidance in relation to VMAT, by means of two-dimensional portal images (Burman et al 2009), pre-treatment kilovoltage CT (Bedford 2008), simultaneous kilovoltage CT (Nakagawa et al 2009) or tomosynthesis (Zhou et al 2009). Megavoltage images acquired during treatment with an electronic portal imaging device (EPID) have been considered as a planar image guidance tool (Liu et al 2009), a geometrical quality-assurance method (Yi et al 2009), or a dose-measurement method in two(Iori et al 2010) or three dimensions (Mans et al 2010).

There is a further interesting possibility. Megavoltage CT (MVCT) reconstruction from portal images is possible (Lewis et al 1992, Mosleh-Shirazi et al 1998) and now a well-developed technology (Pouliot 2007). For such reconstructions, to acquire the necessary data, additional portal images are typically obtained before or after the delivery of a treatment beam. For the case of a VMAT treatment, however, can images acquired by an Electonic Portal Imaging Device (EPID) during the treatment be used for CT reconstruction? In a strict mathematical sense, the answer is: no. In a VMAT delivery, a multi-leaf collimator (MLC) defines a relatively small exposed field. In any plausible delivery the images acquired in the Beam's Eye View (BEV) do not capture the full spatial extent of the patient. The data therefore form an incomplete Radon transform of the subject and exact 'inversion' to reconstruct the patient is impossible. This situation of truncated data has, in the past, been taken as the starting point 
for 'local' (lambda) tomography (Faridani et al 1992). However, radiotherapy treatments using VMAT show a particularly challenging type of data-incompleteness. As the x-ray source rotates, the MLC leaves are in motion. This means that, some points, and perhaps many points, are in the exposed field for only a sub-set of the arc. This leads to possible dataincompleteness in an angular sense. We show, however, regardless, that the application of a cone-beam formula to portal images acquired during a VMAT delivery leads to a readily recognizable CT volume. Further, local (lambda) tomography can be used to enhance the visual quality of the images. We suggest that such VMAT-CT reconstructions could be a useful tool for treatment position verification.

\section{Theory and method}

\subsection{Global and local reconstruction for VMAT-CT}

We will denote a 3D function describing the attenuation coefficient of a subject by the function, $f(r, \phi, z)$, where $(r, \phi, z)$ are cylindrical polar coordinates. In practice, either the Ram-Lak kernel (Ramachandran and Lakshminarayanan 1971) or an apodizing kernel are applied in CT reconstruction. This results in the reconstruction not of $f(r, \phi, z)$ itself, but rather this function smoothed by a point-spread function (PSF). In the case of a Ram-Lak kernel this PSF arises only from the finite pixel-width. In the case of an apodizing kernel, additional smoothing is included to reduce image noise. Consider such a reconstructed "image" in the $(r, \phi)$ plane. The smoothed 3D function, $f_{R}(r, \phi, z)$, can be expressed as,

$$
f_{R}(r, \phi, z)=E_{R}(r) * * f(r, \phi, z),(1)
$$

where $E_{R}(r)$ is a 2D PSF in the $(r, \phi)$ plane and $* *$ represents a $2 \mathrm{D}$ convolution operation. Now consider the cone-beam geometry illustrated in figure 1 (a) and (b). The variables SAD and SDD are the source-to-axis distance and source-to-detector distance, respectively. The variable $\beta$ is the angle of the source with respect to the positive y-axis. The variables $U$ and $V$ denote the position of a point in the imaging plane. The Feldkamp cone-beam reconstruction algorithm (Feldkamp et al 1984) can then be written as,

$$
f_{R}(r, \phi, z)=\frac{1}{2} \int_{0}^{2 \pi} d \beta\left(\frac{\mathrm{SAD}}{\mathrm{SAD}-s}\right)^{2} \int_{-u_{\max }}^{u_{\max }} d u D_{\beta}(u, v) e_{R}\left(u^{\prime}-u\right)\left(\frac{\mathrm{SAD}}{\sqrt{\mathrm{SAD}^{2}+u^{2}+v^{2}}}\right),
$$

where,

$$
\begin{array}{r}
u=U\left(\frac{\mathrm{SAD}}{\mathrm{SDD}}\right), v=V\left(\frac{\mathrm{SAD}}{\mathrm{SDD}}\right), \\
u^{\prime}=t \frac{\mathrm{SAD}}{\mathrm{SAD}-s}(4) \\
t=r \cos (\phi-\beta), s=r \sin (\phi-\beta)
\end{array}
$$

and $D_{\beta}(u, v)$ is the cone-beam ray-projection at the point on the detector defined by $(u, v)$ for the source rotation, $\beta$. The function, $e_{R}(u)$, is related to the PSF and in this work takes the form (see Appendix A),

$$
e_{R}(u) \approx \frac{1}{2 \pi} \Lambda \varepsilon_{R}(u)
$$

where

$$
\begin{aligned}
\varepsilon_{R}(u) & =\frac{1}{R \sqrt{\pi}} \frac{\Gamma\left(\frac{5}{2}+\alpha\right)}{\Gamma(2+\alpha)}\left(1-\left(\frac{u}{R}\right)^{2}\right)^{\alpha+1}, \text { if }|u| \leq R \\
& =0, \quad \text { otherwise }
\end{aligned}
$$

where $R$ defines a range and $\Lambda$ is the Calderon operator (Faridani et al 2000). The Calderon operator is pseudo-differential operator that performs a deblurring operation. Consider a 
Euclidean space $\Re^{n}$ in which a point is defined by the vector, $\underline{r}$. A function in this space, $\varepsilon(\underline{u})$, may have a Fourier transform, $\mathrm{E}(\underline{\rho})$, and a Fourier transform pair,

$$
\mathrm{E}(\underline{\rho})=\mathrm{F}\{\mathcal{\varepsilon}(\underline{u})\}=\int_{\Re^{n}} e^{-2 \pi i \underline{u} \cdot \underline{\rho}} \mathcal{\varepsilon}(\underline{u}) \text { and } \varepsilon(\underline{u})=\mathrm{F}^{-1}\{\mathrm{E}(\underline{\rho})\}=\int_{\Re^{n}} e^{+2 \pi i \underline{u} \cdot \underline{\rho}} \mathrm{E}(\underline{\rho}) .
$$

The Calderon operator, acting on a function $\mathcal{E}(\underline{u})$, is then defined as

$$
\Lambda \varepsilon(\underline{u})=F^{-1}\{2 \pi|\rho| F\{\varepsilon(\underline{u})\}\},
$$

where $\Lambda$ acts on the $n$-dimensional space. In expression (6) the Calderon operator acts on the variable $u$ only (i.e. $n=1$ ). Note that it would also be possible to define $e_{R}(u)$ as a standard global CT convolution kernel, such as a Ram-Lak or Hamming kernel. This would, however, preclude the extension to local (lambda) reconstruction discussed subsequently.

Returning to equation (2), we note that the factors appearing in the round-brackets of this expression are geometric weighting factors. In this work we will assume that SAD $>u, v$ and ignore such factors: this simplifies the resulting formulae (see also Section 2.3). Equation (2), however, is inadequate, as it stands, in dealing with the data insufficiencies in the projection data. We therefore propose the VMAT-CT formula,

$$
f_{R}(r, \phi, z) \approx \frac{1}{2} \frac{\Theta\left(\int_{0}^{2 \pi} M_{\beta}\left(u^{\prime}, v\right) d \beta-\beta^{\prime}\right)}{\frac{1}{2 \pi} \int_{0}^{2 \pi} M_{\beta}\left(u^{\prime}, v\right) d \beta} \int_{0}^{2 \pi}\left[M_{\beta}\left(u^{\prime}, v\right) \int_{-u_{\max }}^{u_{\max }} d u D_{\beta}^{e x t r a p}(u, v) e_{R}\left(u^{\prime}-u\right)\right] d \beta,
$$

where we shall define the extra constituents in detail below. The MLC aperture is described by the masking function, $M_{\beta}(u, v)$. If the aperture extent, at a particular $v$, and $\beta$, is defined by the interval, $u_{1}(v, \beta)<u<u_{2}(v, \beta)$, then

$$
M_{\beta}(u, v)=\begin{array}{ll}
1, & \text { if } u_{1}(v, \beta)<u<u_{2}(v, \beta) . \\
0, & \text { otherwise }
\end{array}
$$

. The acquired ray-projections must be extrapolated for the purposes of the convolution integral in (10). The extrapolation scheme chosen here was:

$$
\begin{array}{rll}
D_{\beta}(u, v), & \text { if } & u_{1}(v, \beta) \leq u \leq u_{2}(v, \beta) \\
D_{\beta}^{\text {extrap }}(u, v)=D_{\beta}\left(u_{1}, v\right), & \text { if } & u<u_{1}(v, \beta) \\
D_{\beta}\left(u_{2}, v\right), & \text { if } & u>u_{2}(v, \beta)
\end{array} .
$$

More sophisticated extrapolation schemes are, of course, possible. This makes no assumption about specific MLC orientation. However, with some collimator rotations (e.g. $90^{\circ}$ ) it would be possible for leaves to split the aperture into more than a single region in the $u$-direction. Such cases (not considered here) would require a slightly more complex masking function and extrapolation. The masking function is included inside the back-projection integral to prevent the back-projection of convolved ray-projections outside of the BEV. We emphasise that we are interested only in visual images, not quantitative densities within the patient, and so are not unduly worried if the data set is not strictly complete over an angular range. However, recognizing that, where the data is woefully insufficient the image quality will suffer, we include a cut-off radian coverage, $\beta$ '. This parameter appears in the Heaviside stepfunction, $\Theta(\ldots)$, of equation (10). The $\Theta$-function forces the reconstructed value to zero at positions in the BEV for less than $\beta$ ' radians. This allows us to eliminate reconstruction points for which so little data are available that they are likely to be misleading. The denominator of the fraction in which the $\Theta$-function appears is present to normalise the contributions according to the angular extent of data available. Thus, for example, a voxel in the BEV for $75 \%$ of the $2 \pi$ arc (i.e. $\frac{1}{2 \pi} \int_{0}^{2 \pi} M_{\beta}\left(u^{\prime}, v\right) d \beta=0.75$ ), will be divided by a factor 0.75 to approximately correct for the missing contributions. 
We have acknowledged that due to data-insufficiencies in the set of projections obtained in a VMAT treatment, exact and unique reconstruction of $f_{R}(r, \phi, z)$ is impossible. The datainsufficiency in the VMAT-CT problem, in particular, the width truncation of projections by the MLC, suggests the possibility of using local tomography techniques (Faridani et al 1992). The idea of local (lambda) CT is not to reconstruct $f_{R}(r, \phi, z)$ but rather a related object,

$$
\Lambda^{\mathrm{m}} f_{R}(r, \phi, z)
$$

where $m$ is an integer denoting the number of repeat operations of the Calderon operator. We note that in (13), $\Lambda$ acts on the $n=2$ space of $(r, \phi)$ (but not over $z$ ). The new object (13) has many of the same properties as the original attenuation coefficient function (Faridani et al 1997). The generalised version of the VMAT-CT reconstruction formula we propose is then,

$$
\Lambda^{m} f_{R}(r, \phi, z) \approx \frac{1}{2} \frac{\Theta\left(\int_{0}^{2 \pi} M_{\beta}\left(u^{\prime}, v\right) d \beta-\beta^{\prime}\right)}{\frac{1}{2 \pi} \int_{0}^{2 \pi} M_{\beta}\left(u^{\prime}, v\right) d \beta} \int_{0}^{2 \pi}\left[M_{\beta}\left(u^{\prime}, v\right) \int_{-u_{\max }}^{u_{\max }} d u D_{\beta}^{\text {extrap }}(u, v) e_{R}^{(m)}\left(u^{\prime}-u\right)\right] d \beta \text {, }
$$

where the generalized function $e_{R}^{(m)}(u)$ is (see Appendix A)

$$
e_{R}^{(m)}(u) \approx \frac{1}{2 \pi} \Lambda^{m+1} \varepsilon_{R}(u)
$$

What are the potential benefits of extending tomographic reconstruction beyond the "normal" $m=0$ case? If $m$ is even, such as the $m=0$ case, $e_{R}^{(m)}(u)$ has a non-compact support and is termed "global". The convolved ray-projection, to be back-projected, then depends on every unconvolved ray-projection along the $u$ integration direction, for fixed values of $v$. Therefore, if the patient does not entirely fit within the field-of-view at any angle $\beta$, required data are absent. Despite this, in some cases, the ray-projections through the patient outside the acquired set can be extrapolated reliably enough to provide an acceptable reconstruction (e.g. Ohnesorge et al 2000). It can be shown, however, that if $m \geq-1$ and odd then $e_{R}^{(m)}(u)$ can have a compact support and be described as "local". This means that a convolved rayprojection, to be back-projected, depends only on the unconvolved ray-projections in its local vicinity (within a distance $R$ in fact). Thus the $m=1$ algorithm has less restrictive data requirements than the $m=0$ case. In some cases therefore we might expect local tomography $(m=1)$ to provide more useful information than the global $(m=0)$ algorithm (Anastasio et al 2003).

To encapsulate the benefits of either approach (local or global tomography) we propose a hybrid semi-local construction,

$$
\Sigma_{\sigma} f_{R}(r, \phi, z)=f_{R}(r, \phi, z)+\sigma R \Lambda f_{R}(r, \phi, z),
$$

where $\sigma$ is a dimensionless parameter and $R$ is again the range parameter. 
(a) The $X$-Y plane

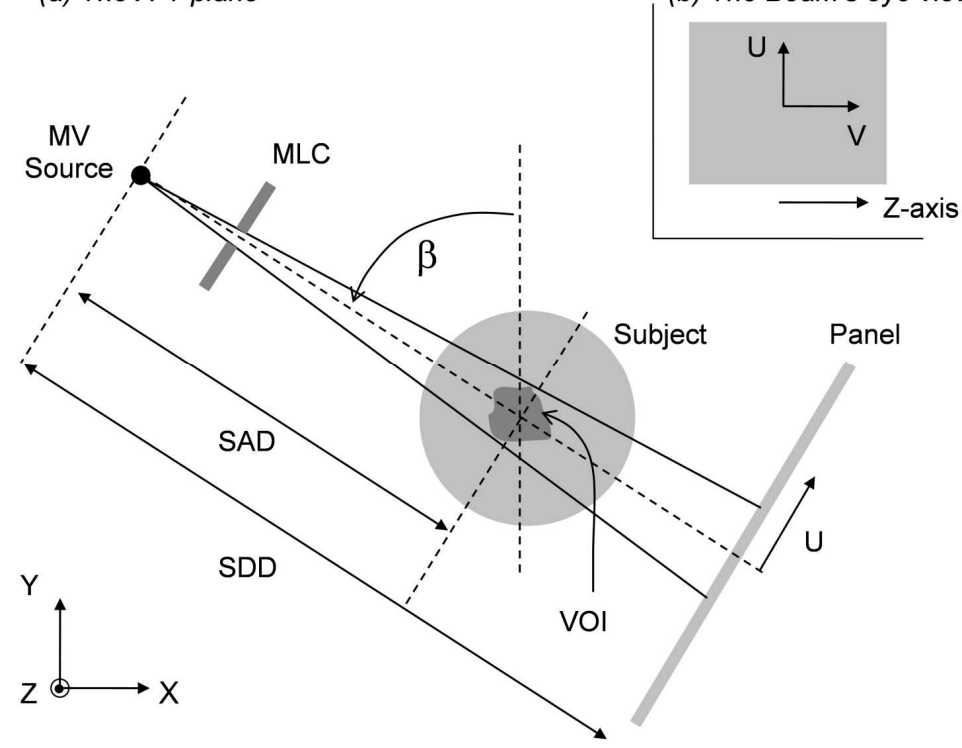

Figure 1: The cone-beam geometry of the VMAT portal image acquisition. The Volume-ofInterest (VOI) to reconstruction is shown.

\subsection{Data acquisition for VMAT-CT}

Three images sets have been considered to demonstrate the feasibility of this method of reconstruction. Two were produced using a Rando anthropomorphic phantom (The Phantom Laboratory, Salem NY, USA), while one was a clinical treatment plan on a patient. Phantom plans were produced using the SmartArc algorithm on Pinnacle, version 9 (Philips Radiation Oncology Systems, Madison, USA). The collimator rotation angle was set to a nominal value of $180^{\circ}$. Two different volumes were planned: first, a small and simple treatment of the antrum sinus to a single dose level of 65 Gy in 30 fractions; and second, a more complex treatment of the oropharynx, with the primary tumour and involved unilateral nodes prescribed to $65 \mathrm{~Gy}$ in 30 fractions, and elective nodes to $54 \mathrm{~Gy}$ in 30 fractions concurrently. Both tumour sites were outlined for planning in accordance with clinical practice and the plans satisfied the standard clinical planning constraints in our institution. The patient had a carcinoma of the oesophagus, and was optimised using an in-house planning system, Autobeam, and calculated for clinical use on Pinnacle using a static beam approximation (Bedford 2009). The collimator rotation angle was set to $5^{\circ}$. The planned dose was $54 \mathrm{~Gy}$ in 30 fractions. In all cases, the planning goal was to cover the planning target volume (PTV) with $95 \%$ of the prescribed dose, while the (principal) prescription was delivered to the mean PTV dose.

The plans were delivered using an Elekta Synergy accelerator, operating at $6 \mathrm{MV}$ and fitted with an MLCi multi-leaf collimator. The MLCi possesses 80-leaves projecting to $1 \mathrm{~cm}$ at the isocentre, and associated with this MLC there are two sets of moveable jaws. Images were acquired using the Elekta iView software in movie mode. Such movies are limited by the software to 256 images, so it was necessary to use frame averaging to allow an entire treatment to be imaged. In these cases, two frames (approximately $450 \mathrm{~ms}$ each) were averaged for the antrum and oesophagus cases, resulting in 151 and 135 images respectively, while for the oropharynx, four-frame averaging was required, resulting in 194 images. Each image is linked to a field in the iView database which gives the time of the start of each image, relative to the point when the panel detected radiation and the first image was started.

In order to obtain the projection angles and other linac data, WinDump (www.winpcap.org) was used to provide the raw data sent from the linac to the iView control computer using the 
proprietary iCOM interface. These data are in plain text format and can be easily converted to a set of times and measured positions for gantry and collimator angles, MLC and jaw positions, and monitor units. The times in the iCOM communications are truncated to a resolution of only $1 \mathrm{~s}$, so the iView computer times (with a resolution of $1 \mu \mathrm{s}$ ) for the receipt of each packet were used. The conversion of the iCOM text stream to meaningful data was implemented using IDL (ITT Visual Information Solutions, Boulder, USA).

The absolute times in the iView database were not found to relate well to the packet-receipt times, so a delay (conceptually identical to the packet transit time) was introduced. This delay was found by maximising the product-moment correlation coefficient, $r$, of the inverse pixel factor (PF) (proportional to the absolute brightness of the brightest point in each image) to the number of MUs delivered during the image integration, on the assumption that the maximum transmission through the treatment portal is approximately constant for all angles. To verify the assignment of gantry angle to image, a phantom was imaged using the plan for the oesophagus treatment. The phantom consisted of two metal bars placed on the treatment couch at the isocentre height, running in the $\mathrm{Z}$ direction, separated by $20 \mathrm{~cm}$. The images closest to gantry $90^{\circ}$ and $270^{\circ}$ where the two bars were closest to superimposition were identified, and the apparent gantry angle calculated from a measurement of the separation in the $U$-direction. This angle was then compared to the angle obtained from the iCOM data with fitted delay.

\subsection{Implementation of VMAT-CT}

The geometry of the planar image-acquisition discussed above consisted of a nominal SAD of $100 \mathrm{~cm}$ and a nominal SDD of $159 \mathrm{~cm}$. The portal imaging device used for acquiring VMATCT projection images was a Perkin Elmer (Massachusetts, USA) amorphous silicon flatpanel detector: this is the standard EPID for an Elekta linac (Elekta Limited, Crawley, UK). The active region of the square detector is of width $W=41 \mathrm{~cm}$ and the panel consists of $1024 \times 1024$ pixels. Therefore, it follows that: $u_{\max }=(W / 2)(\mathrm{SAD} / \mathrm{SDD})=12.9 \mathrm{~cm}$, assuming a centred panel. In this study the signal was binned down to $512 \times 512$ pixels. A five-pixel median filter was also applied to the acquired projections to reduce noise. The digital-values (DVs) associated with the pixels are of 16-bit depth (i.e. take values from 0 to $2^{16}-1$ ). Each portal image consisted of an array of digital-values, DV $[i, j]$, where $i$ and $j$ denote the pixel number in the $u$ and $v$ directions, respectively. The energy deposited in the detector can be (approximately) related to the $D V$ by,

$$
E[i, j]=\operatorname{constant} \times \frac{2^{16}-1-\mathrm{DV}[i, j]}{\mathrm{PF}},
$$

where PF is the pixel normalisation factor produced by the EPID system. The PF will, in general, vary between individual portal images. The value of the "constant" in (17) will depend on the energy-response of the panel and the x-ray quality of the beam. Using equation (12) we can choose to normalize the projection data such that,

$$
D_{\beta}[i, j]=\frac{2^{16}-1-\mathrm{DV}[i, j]}{\operatorname{PF}(\beta) \operatorname{MU}(\beta)} \cdot \frac{\min _{\{\text {all } \beta\}}(\operatorname{PF}(\beta) \mathrm{MU}(\beta))}{2^{16}-1} .
$$

where $\operatorname{MU}(\beta)$ is the monitor units (MU) delivered during the acquisition of a portal image at (average) source-angle, $\beta$. We note that this is not the normalization that would correspond to division by an open field. Rather, it removes the variability due to the particular PF and MU associated with a portal image. Since the fluence delivery rate may change continuously during a VMAT delivery, the MU may vary markedly from frame to frame. To account for this, procedures to estimate the total MU for each portal image, such as are outlined in the previous section, are required. Assuming a correctly performed normalization, the varying fluence delivery rate will not have an affect on the reconstructed images, apart from, of course, its impact on reconstruction noise. Since we are not normalizing by the open-field, the reconstructed quantity cannot be expected to accurately provide values of $\Sigma_{\sigma} f_{R}(r, \phi)$. 
However, as previously stated, we are interested in producing useful images, not quantitative voxel values.

An EPID masking function (EPID-MF) was defined as a binary image of a portal image: value 1 for an exposed pixel and 0 for a pixel in the shadow of a leaf. A gradient-based edgedetection was used to detect the aperture formed by the MLC leaves in each portal image and hence assign binary values. Further, for each portal image, a binary MLC masking function (MLC-MF) was constructed, using linac readout of average leaf-position during each portal image exposure and the known collimator rotation. The matching of the EPID-MF to the MLC-MF allowed the extraction of the radiation isocentre and approximate correction for gantry flex. A downhill simplex algorithm was implemented in Fortran 95 (Numerical Recipes in Fortran 90: Press et al 2002) for this purpose, using a least-squares cost-function. The pre-processing and reconstruction steps were also performed using custom written Fortran 95 executables. The whole reconstruction and image manipulation process was managed with scripts written in the Python 2.6 language. Reconstruction was carried out using equations (3-7, 11-16, 17-18).

Convolution operations, including the application of the Calderon operation, were performed using Fast Fourier Transforms (FFTs) and multiplication in Fourier space. We note that we can express (15) in terms of the standard Ram-Lak kernel i.e.

$$
e_{R}^{(m)}(u)=\frac{1}{2 \pi}\left[2 \pi \kappa_{R L}(u) *\right]^{m+1} \varepsilon_{R}(u),
$$

where * denotes a 1D convolution and $\kappa_{R L}(u)$ is the Ram-Lak kernel (Kak and Slaney, 2001). Making use of the convolution theorem, the FFT of $\varepsilon_{R}(u)$ was multiplied by the FFT of the Ram-Lak kernel $m+1$ times to provide the FFT of $e_{R}^{(m)}(u)$. We further note that we can make the replacement

$$
\Lambda^{m} f_{R}(r, \phi, z) \rightarrow \Sigma_{\sigma} f_{R}(r, \phi, z),
$$

in equation (10) with the simple kernel substitution,

$$
e_{R}^{(m)}(u) \rightarrow e_{R}^{(0)}(u)+\sigma R e_{R}^{(1)}(u)=\left[\delta(u)+2 \pi \sigma R \kappa_{R L}(u)\right] * e_{R}^{(0)}(u) .
$$

This trick saves the computational complexity of conducting two repeat filtered backprojections over the reconstruction volume and was used in this work. This approach does, however, suffer the drawback of not allowing a posteriori alteration of the parameter $\sigma$, following the back-projection. Such a substitution in the filter would also have been impossible, had we not assumed $\mathrm{SAD}>>u, v$, which eliminated the presence of spatially varying weighting factors (see Appendix A).

\section{Results}

As illustrations of the VMAT treatment plans, figures 2 shows, with isodose lines, axial slices (parts (a), (c) and (e)) and sagittal slices (parts (b), (d) and (f)) of the planning CT scans for the antrum, oropharynx and oesophagus treatments. Note that the antrum and oesophagus cases, as fairly simple treatments, have associated generally convex isodose lines. The oropharynx treatment, on the other hand, as a quite complex head and neck plan, shows more irregular isodose lines. In particular, note the concavities in the isodose lines of figure 2 (c), where these lines curve around to avoid the spinal cord.

Figures 3 (a) to (f) illustrate portal images acquired at gantry angles of $\sim 15^{\circ}$ (parts (a), (c) and (e)) and $\sim 105^{\circ}$ (parts (b), (d) and (f)) for the antrum, oropharynx and oesophagus patient treatments. The individual portal images are each $41 \times 41 \mathrm{~cm}^{2}$ in area. The antrum treatment images, (a) and (b), show small, generally convex treatment apertures that change relatively slowly with gantry angle. The oropharynx treatment images, (c) and (d), show far more irregular and rapidly changing apertures. The blur due to leaf movement during portal image acquisition is discernable. Figures 2 (e) and (f) show two portal images for the oesophagus 
treatment. In this case, the apertures are larger and more slowly varying than in the oropharynx case, but still exhibit irregular field shapes.

Figure 4 shows the correlation coefficient, $r$, between the reciprocal of the pixel factor and MU for varying time offset, for the oesophagus treatment. A clear peak is seen. Using this method, as described in Section 2.2, the angular difference between the angle inferred from the iCOM-captured data and the angles extracted from the images was less than $0.3^{\circ}$ at the two near-horizontal angles measured. This validated the method of inferring the time delay offset.

Figure 5 (a), as an illustrative example, shows the determinations of the $U$-displacement for each portal image of the antrum treatment. The displacement of the centre of the EPID panel from the radiation isocentre was calculated as described above in Section 2.2. The uncertainties in matching the EPID-MFs and MLC-MFs in the $U$-direction are such that any trend over angle is obscured. Therefore a constant displacement was calculated from the mean of all determinations, as shown by the flat line in the figure. The standard deviation (SD) about this line was $1.7 \mathrm{~mm}$. The uncertainty in the matching of the apertures is primarily due to the beam penumbra and leaf movement during acquisition. The random spread of the determinations in the $V$-direction is smaller, allowing a trend with gantry angle to be identified. A sinusoidal function provided a satisfactory fit, as illustrated in figure 5 (b). The $\mathrm{SD}$ about this trend was $1.4 \mathrm{~mm}$. Since the collimator angle in this case was nominally $180^{\circ}$ the leaf movement is completely along the $U$-direction. Projecting to the isocentre using equations (3), the uncertainty (SD) in the location of the isocentre, as determined from an individual projection, becomes $\sim 1 \mathrm{~mm}$ in the $u$ and $v$ directions. We note that this does not include any uncertainties arising from an imperfect calibration of jaw and leaf positions, which are of the same order in their magnitude.

In figure 6 (a), a region of the mediastinum of the oesophagus patient is shown, from the planning CT scan. The global and local CT reconstructions of this region from VMAT-CT images projection are shown in figure 6 parts (b) - (d). In all cases the parameter $\beta$ was set to 3.4 radians $\left(\pi+\right.$ twice the maximum fan-angle). Figures 6 (b) and (c) show the global $\left(f_{R}\right.$; $\left.S_{f}=2.0\right)$ and local $\left(\Lambda f_{R} ; S_{f}=4.0\right)$ images, respectively. The edges in the global image are markedly less well-defined. However, the edge-enhancement in the local images leads to a visible mottling. Figure 6 (d) shows the semi-local image ( $\Sigma_{\sigma} f_{R} ; \sigma=0.5 ; S_{f}=4.0$ ), which is a compromise between the extremes of images (b) and (c). Note that there is no reason that we should expect a correspondence in detail between figures 6 (a) and the parts (b) to (d). In particular note that: (i) in the time (i.e. weeks) between the planning scan and the VMAT fraction, changes in anatomy may have occurred, (ii) respiratory motion occurs in this region, (iii) the axial resolution of the planning scan is lower than for the VMAT-CT reconstruction and (iv) the images have not been accurately registered (small rotations as well as translations may be present). None-the-less a general gross correspondence is suggested.

Axial, sagittal and coronal VMAT-CT reconstructions are shown in figure 7 (a), (c) and (e), respectively, for the treatment to the antrum of a Rando phantom $\left(\Sigma_{\sigma} f_{R} ; \sigma=0.5\right.$; $S_{f}=4.0$ ). For comparison, closely corresponding slices are shown from the planning CT scan, in figures 7 (b), (d) and (f). Planning target volume (PTV) contours are depicted in the planning CT slices. The anatomical structures of the antrum are surprisingly distinguishable in the VMAT-CT images, despite the image quality being inferior to that in diagnostic CT. Again, $\beta$ was set to 3.4 radians.

Due to the greater leaf movement and more irregular field shapes, the angular data insufficiency at each reconstruction point was, in general, greater for the oropharynx case. This necessitated the lowering of the $\beta$ parameter. This was set to 1.57 radians $\left(90^{\circ}\right)$. Figures 
8 (a), (c) and (e) show axial, sagittal and coronal VMAT-CT reconstructions, respectively, for the oropharynx treatment to a Rando phantom $\left(\Sigma_{\sigma} f_{R} ; \sigma=0.5 ; S_{f}=4.0\right)$. Comparison slices are shown from the planning CT scan in figures 8 (b), (d) and (f), along with the associated PTV contours. The air cavities within the patient are still quite distinguishable and the narrow gaps between the slabs of the Rando phantom are observable in the sagittal and coronal slices. Note that the axial plane, as shown in figure 8 (a), is most severely affected by the angular data insufficiency, although structures are still discernable. Note also, that structures that are protected within the treatment, such as the spinal column, are missing from the reconstruction, as would be expected.

The final image set reconstructed was for that of a patient receiving treatment in the oesophagus region. Axial, sagittal and coronal VMAT-CT reconstructions are shown in figure 9 (a), (c) and (e), respectively $\left(\Sigma_{\sigma} f ; \sigma=0.5 ; S_{f}=4.0\right)$. Corresponding slices, with associated PTV contours, are shown from the planning CT scan in figures 9 (b), (d) and (f). Again, the anatomical features of the thorax are remarkably identifiable and match well with the planning CT scan. The aperture sizes for this treatment were quite large, compared to the antrum case, but without the degree of leaf-modulation present in the oropharynx case. For these reasons the quality of the reconstructions for the oesophagus is perhaps the best of the three examples.

(a)

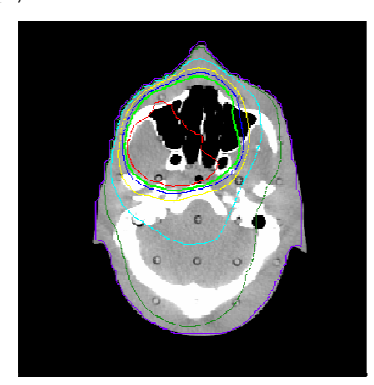

(c)

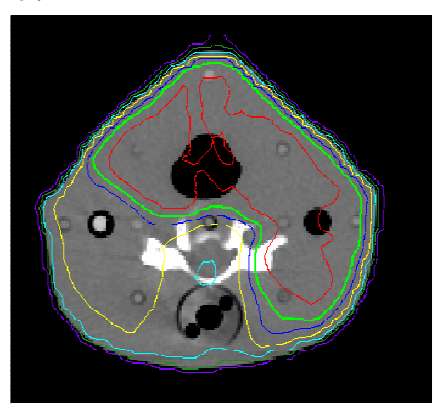

(e)

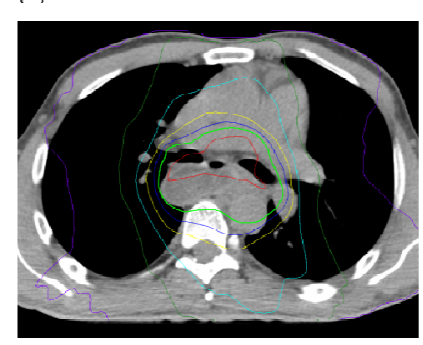

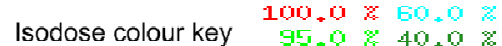
$90.0 \& 20.02$

(b)

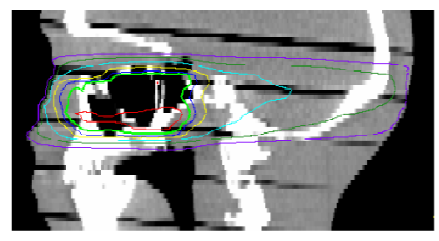

(d)

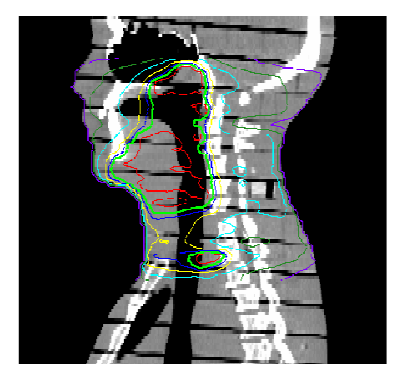

(f)

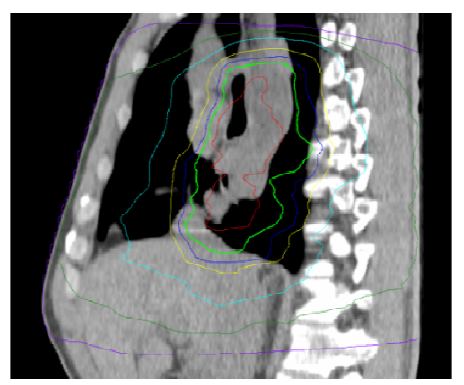

Figure 2. Example axial planning CT slices with planned isodose lines for: (a) antrum treatment (axial slice), (b) antrum treatment (sagittal slice), (c) oropharynx treatment (axial slice), (d) oropharynx treatment (sagittal slice), (e) oesophagus treatment (axial slice) and (f) oesophagus treatment (sagittal slice). 


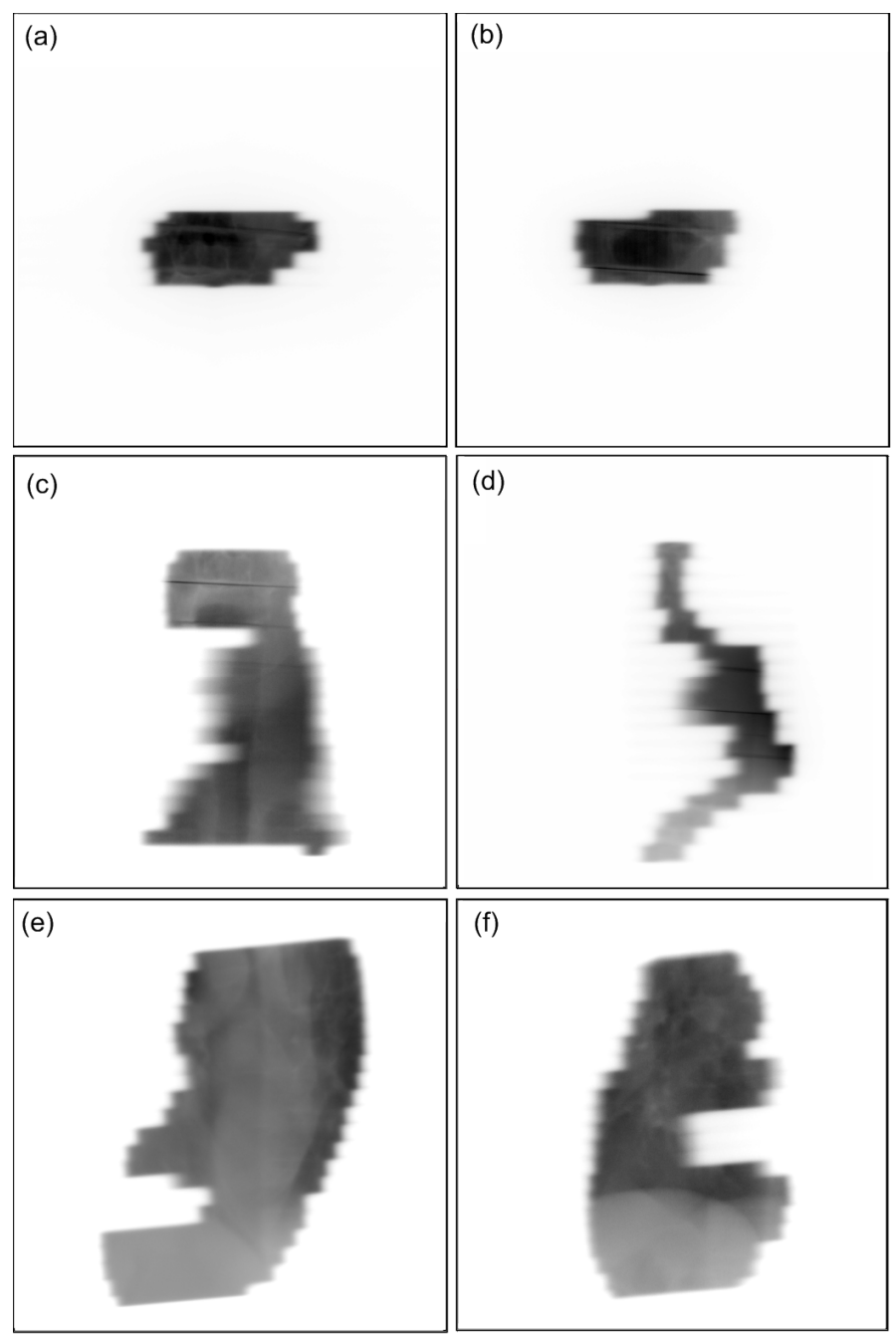

Figure 3. Treatment portal images for: (a) Antrum treatment (gantry angle $\sim 15^{\circ}$ ), (b) antrum treatment (gantry angle $\sim 105^{\circ}$ ), (c) oropharynx treatment (gantry angle $\sim 15^{\circ}$ ), (d) oropharynx treatment (gantry angle $\sim 105^{\circ}$ ), (e) oesophagus treatment (gantry angle $\sim 15^{\circ}$ ) and (f) oesophagus treatment (gantry angle $\sim 105^{\circ}$ ).

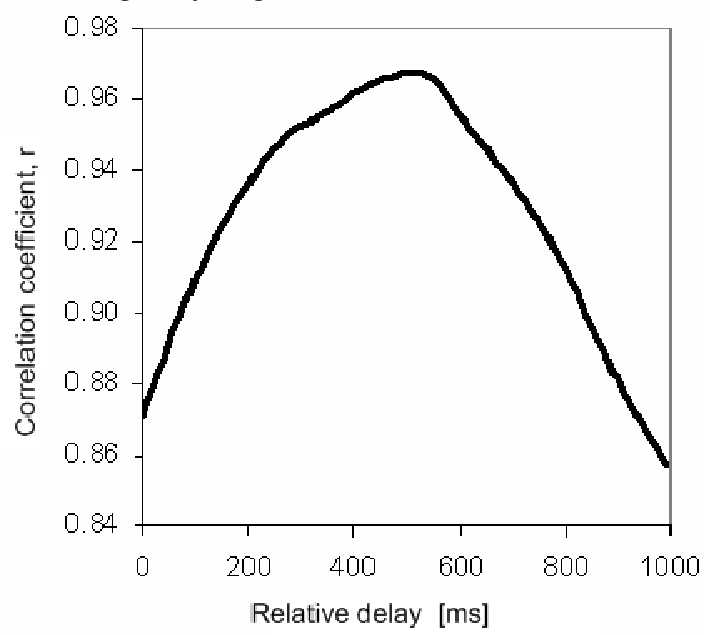

Figure 4. Calculated product-moment correlation coefficient, $r$, between inverse pixel factor and MU, plotted against relative time delay. 

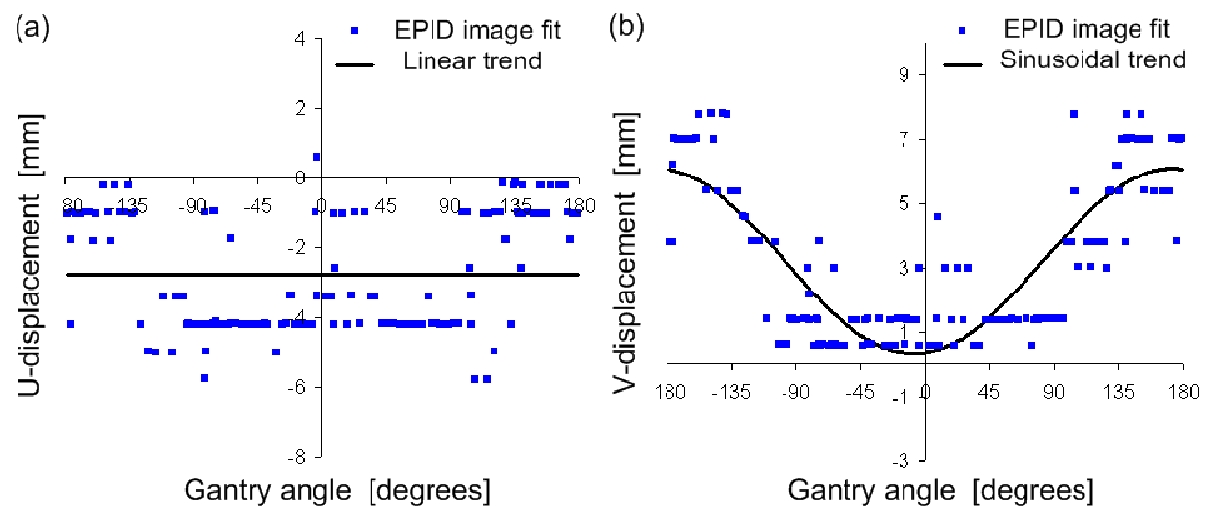

Figure 5. Position-maps of the displacement of the centre of the flat-panel detector from the projected isocentre position for: (a) the $U$-direction and (b) the $V$-direction.
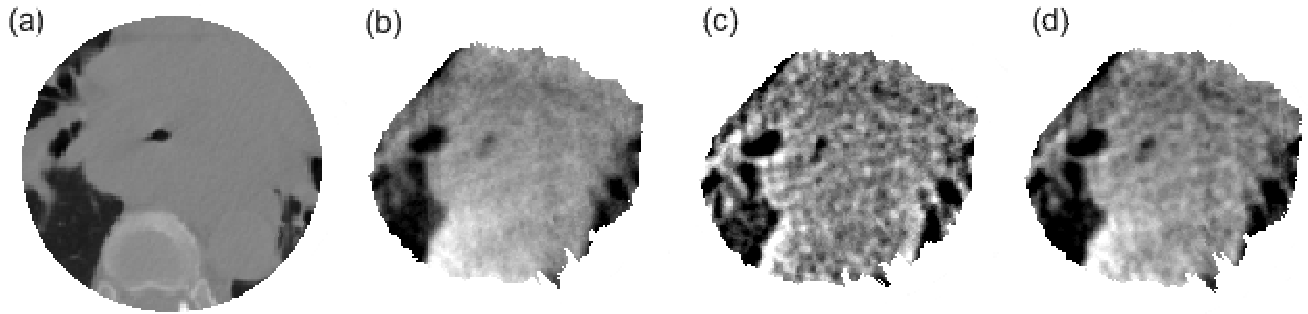

Figure 6. Axial slices reconstructed through the mediastinum of the oesophagus patient for: (a) planning CT, (b) VMAT-CT $\left(f_{R}\right)$, (b) VMAT-CT $\left(\Lambda f_{R}\right)$ and (d) VMAT-CT ( $\left.\Sigma_{\sigma=0.5} f_{R}\right)$.

(a)

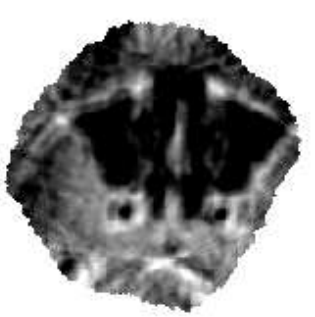

(c)

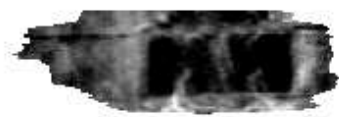

(e)

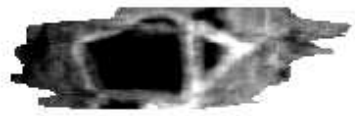

(b)

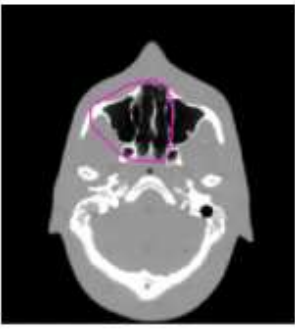

(d)

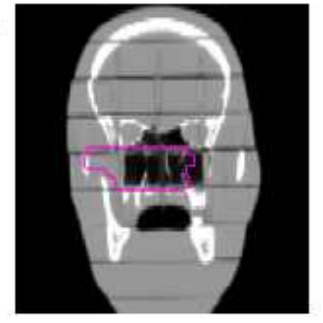

(f)

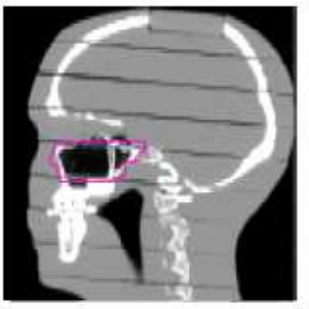

Figure 7. Reconstructed VMAT-CT slices $\left(\Sigma_{\sigma=0.5} f_{R}\right)$ for the antrum treatment in the (a) axial, (c) sagittal and (e) coronal planes. The corresponding planning CT slices, along with PTV contours, sare shown in the (b) axial, (e) sagittal and (f) coronal slices. 

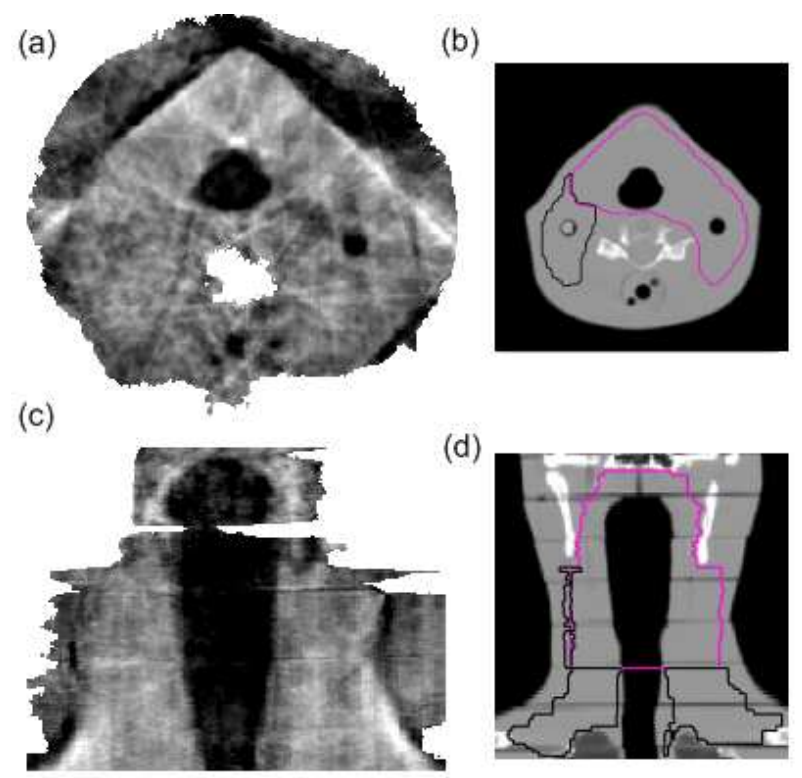

(d)

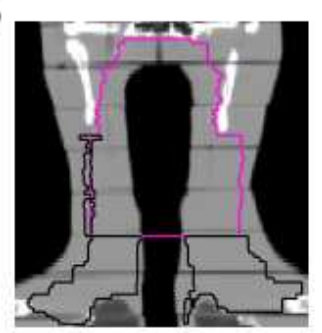

(e)

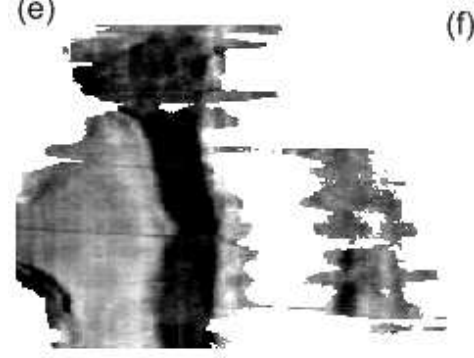

(f)

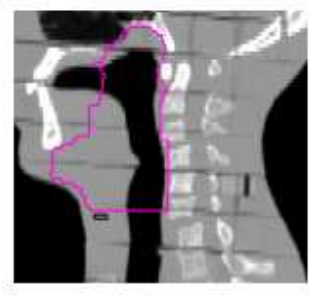

Figure 8. Reconstructed VMAT-CT slices $\left(\Sigma_{\sigma=0.5} f_{R}\right)$ for the oropharynx treatment in the (a) axial, (c) sagittal and (e) coronal planes. The corresponding planning CT slices, along with PTV contours, are shown in the (b) axial, (e) sagittal and (f) coronal slices. 
(a)

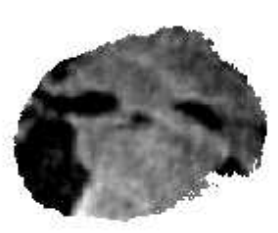

(c)

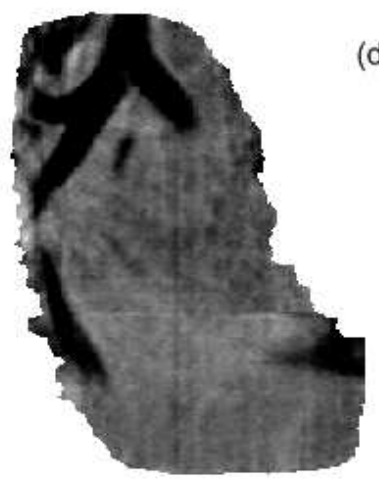

(e)

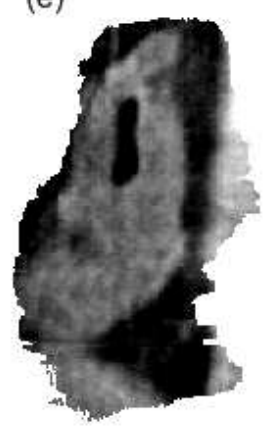

(b)

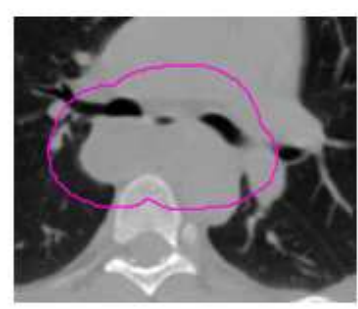

(d)

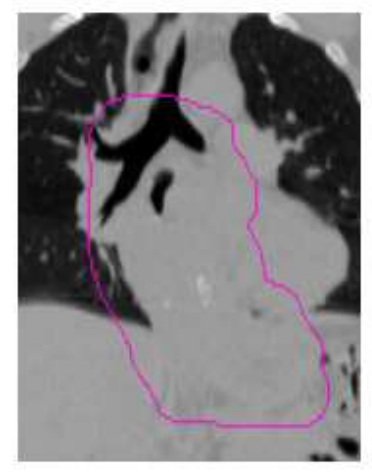

(f)

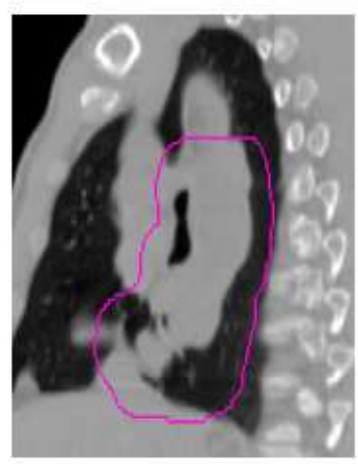

Figure 9. Reconstructed VMAT-CT slices $\left(\Sigma_{\sigma=0.5} f_{R}\right)$ for the oesophagus treatment in the (a) axial, (c) sagittal and (e) coronal planes. The corresponding planning CT slices, along with PTV contours, are shown in the (b) axial, (e) sagittal and (f) coronal slices.

\section{Discussion}

It cannot be disputed that an exact and unique cone-beam reconstruction from portal images acquired in a VMAT delivery is impossible for to a number of reasons. Two important ones in the context of this work are:

1) Severe width-truncation of the portal images;

2) Insufficiency in angular coverage of reconstruction points.

Yet, in this paper, we have demonstrated that recognizable images can still be reconstructed from the VMAT portal projections (figures 7 (a)-(c), figures 8 (a)-(c) and figures 9 (a)-(c)). The width-truncation can be dealt with by extrapolation e.g. equation (8). Errors from this extrapolation largely affect the low-spatial frequency content of the image. This fact allows many features to still be distinguished, even for CT algorithms that are, technically, non-local (e.g. $m=0$ case). The use of local CT algorithms (e.g. $m=1$ case) offer some potential advantages. The definition of boundaries is somewhat improved at the price of the introduction of mottling (compare figures 6 (a) and (b)). It is perhaps surprising, despite the theoretical advantages, that the local algorithm implemented here did not provide a dramatic improvement over the global reconstruction. On the other hand, the fact that the convolution kernel $e_{R}(u)$ is non-compact does not prevent the contributions predominately coming from a 
local region. The problem of the insufficiency of angular coverage, however, can result in dramatic distortions of the reconstructed image, as seen, for example, in the axial slice depicted in figure 9 (a). In all the examples investigated here, the data collected during a VMAT treatment allowed recognisable features to be reconstructed. No doubt improved algorithms and methods can be developed. The advantage of the filtered back-projection approach is its simplicity, speed and robustness. However, for example, in the past, iterative reconstruction methods have proved very successful in dealing with incomplete data sets (Snyder et al 2006). The inclusion of prior information, for example, from a planning CT scan of the same subject, may also provide the potential for improved reconstructions. We hope that this preliminary work will provide an impetus for such improvements. We note that, currently, it is difficult to be certain whether a discrepancy between a VMAT-CT reconstruction and a planning scan is due to changes in anatomy, motion (e.g. respiration) or due to reconstruction artefacts. This problem is an issue in all reconstruction method, more notably with those with data insufficiencies such as the Feldkamp CBCT algorithm, and is perhaps a more severe problem for the VMAT-CT algorithm developed here. Such questions of image quality and veracity can only be answered with further patient and phantom studies. The relationship between treatment complexity and objective measures of VMAT-CT image quality therefore remains an open question, to be investigated in future work, along with the relative advantages of global and (semi-)local algorithms.

None-the-less VMAT-CT is potentially a powerful tool for position verification during radiotherapy treatment. The radiation isocentre has been identified on the image by means of the accelerator's measured leaf and jaw positions. The displacement from this isocentre to the planned isocentre can easily be obtained by fusing the resulting CT images with the planning CT. An attractive feature of this method is that the isocentre position is determined without any knowledge of the plan itself, only of the delivery, which makes it procedurally the equivalent of a treatment-time cone-beam CT. The quantitative evaluation of the feasibility of image registration for such position verification requires further study.

\section{Conclusion}

Tomographic reconstructions of surprisingly good visual quality are possible from portal images acquired during a VMAT radiotherapy treatment fraction. Such VMAT-CT reconstructions may provide a useful tool for treatment position verification. Additionally, the techniques of local-tomography can be used to improve the definition of density changes in the reconstructed images.

\section{Appendix A}

We define the PSF used in this work as identical to that of Faridani et al (1992):

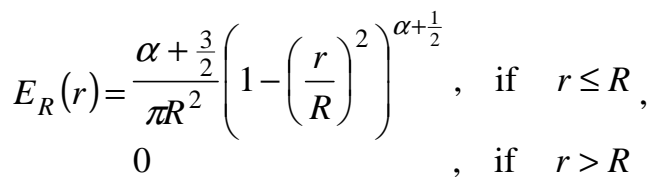

where $\alpha=11.4174$ and $R$ defines support of the PSF. The parameter $R$ is defined as,

$$
R=\sqrt{\frac{2 \alpha+1}{3}} \tau S_{f},
$$

where $\tau$ is the detector pixel pitch and $S_{f}$ is a scaling factor using to tune the smoothing in the image. This PSF is angularly symmetric (i.e. independent of $\phi$ ) has a compact support and its first derivative is zero at the boundary points $r=0$ and $r=R$. Now we provide the definition,

$$
e_{R}^{(m)}(l)=\frac{1}{2 \pi} \Lambda^{m+1} P_{\theta} E_{R}(r),
$$


where $P_{\theta}$ denotes the (parallel-beam) Radon transform operation and $l=x \cos \theta+y \sin \theta$ (where $x=r \cos \phi$ and $y=r \sin \phi$ ). The Calderon operator acts on the variable $l$ only $(n=1)$. It can be shown that, inputting (A.1) gives,

$$
\begin{gathered}
\varepsilon_{R}(u) \equiv P_{\theta} E_{R}(r)= \\
\frac{\alpha+\frac{3}{2}}{\pi R^{2}} \int_{-\sqrt{R^{2}-l^{2}}}^{\sqrt{R^{2}-l^{2}}} d y\left(1-\left(\frac{l^{2}+y^{2}}{R}\right)\right)^{\alpha+\frac{1}{2}}=\frac{1}{R \sqrt{\pi}} \frac{\Gamma\left(\frac{5}{2}+\alpha\right)}{\Gamma(2+\alpha)}\left(1-\left(\frac{l}{R}\right)^{2}\right)^{\alpha+1}, \quad \text { if } \quad l \leq R
\end{gathered}
$$

The function (A.4) needs to be translated from the parallel-beam variable $l$ to the cone-beam variable $u$. It turns out that this is accomplished via the relation (Kak and Slaney 2001, Anastasio et al 2003),

$$
\begin{aligned}
& e_{R}^{(m)}\left(l^{\prime}-l\right)=\left(\frac{\mathrm{SAD}}{\mathrm{SAD}-s} \frac{\sqrt{\mathrm{SAD}^{2}+u^{2}+v^{2}}}{\sqrt{\mathrm{SAD}^{2}+v^{2}}}\right)^{2+m} e_{R}^{(m)}\left(u^{\prime}-u\right), \\
& \approx e_{R}^{(m)}\left(u^{\prime}-u\right)
\end{aligned}
$$

where we have assumed that $\mathrm{SAD}>>u, v$. It therefore directly follows from (A.4) and (A.5), that

where we define,

$$
e_{R}^{(m)}(u) \approx \frac{1}{2 \pi} \Lambda^{m+1} \varepsilon_{R}(u)
$$

$$
\begin{aligned}
\varepsilon_{R}(u) & =\frac{1}{R \sqrt{\pi}} \frac{\Gamma\left(\frac{5}{2}+\alpha\right)}{\Gamma(2+\alpha)}\left(1-\left(\frac{u}{R}\right)^{2}\right)^{\alpha+1}, \text { if }|u| \leq R \\
& =0, \quad \text { otherwise }
\end{aligned}
$$

The Calderon operator in this case, operates over $u$ (i.e. $n=1$ ).

\section{Acknowledgements}

This work was partially supported by research grant C46/A2131 from Cancer Research UK. We acknowledge NIHR funding to the NHS Biomedical Research Centre.

\section{References}

Anastasio M A, Shi D X, Pan X C, Pelizzari CA and Munro P 2003 A preliminary investigation of local tomography for megavoltage CT imaging Med. Phys. 30(11) 2969-2980.

Bedford J 2009 Treatment planning for volumetric modulated arc therapy Med. Phys. 36(11) 5128-5138.

Bedford J L, Nordmark Hansen V, McNair H A, Aitken A H, Brock J E, Warrington A P and Brada M 2008 Treatment of lung cancer using volumetric modulated arc therapy and image guidance: a case study Acta Oncol. 47(7) 1438-43.

Bortfeld T and Webb S 2009 Single-arc IMRT? Phys. Med. Biol. 54 N9-N20.

Burman C, Li J and Chan M 2009 TH-D-210A-02: Portal Imaging for Volumetric Modulated Arc Therapy Med. Phys. 362818.

Faridani A, Buglione K A, Huabsomboon P, Iancu O D and McGrath J 2000 Introduction to Local Tomography Radon Transforms and Tomography (Contemporary Mathematics, Vol. 278, American Mathematical Society), ed E T Quinto et al (Providence, Rhode Island) pp. 29-47.

Faridani A, Finch D V, Ritman E L and Smith K T Local Tomography II SIAM J. Appl. Math. 57(4) 1095-1127.

Faridani A, Ritman E L and Smith K T 1992 Local Tomography SIAM J. Appl. Math. 52(2) 459-484.

Feldkamp L A, Davis L C and Kress J W 1984 Practical cone-beam algorithm J. Opt. Soc. 
Am. A1 612-19.

Kak A C and Slaney M 2001 Chapter 3: Algorithms for reconstruction with nondiffracting sources Principles of Computerized Tomographic Imaging (SIAM) (Philadelphia, USA) pp.49-112.

Iori M, Cagni E, Paiusco M, Munro P, Nahum A 2010 Dosimetric verification of IMAT delivery with a conventional EPID system and a commercial portal dose image prediction tool Med. Phys. 37 377-390.

Lewis D G, Swindell W, Morton E J, Evans P M and Xiao Z R 1992 A megavoltage CT scanner for radiotherapy verification Phys. Med. Biol. 37(10) 1985-99.

Liu W, Wiersma R, Luxton G and Xing L 2009 TH-C-303A-03: Real-Time Motion Detection of Prostate Target During Volumetric Arc Therapy Using Onboard Imaging Devices Med. Phys. 362800.

Mans A, Remeijer P, Olaciregui-Ruiz I, Wendling M, Sonke J-J, Mijnheer B, van Herk M and Stroom J C 2010 3D Dosimetric verification of volumetric-modulated arc therapy by portal dosimetry Radiother Oncol. 94 181-7.

Mosleh-Shirazi M A, Evans P M, Swindell W, Webb S and Partridge M 1998 A cone-beam megavoltage CT scanner for treatment verification in conformal radiotherapy Radiother. Oncol. 48(3) 319-28.

Pouliot J 2007 Megavoltage imaging, megavoltage cone beam CT and dose-guided radiation therapy Front. Radiat. Ther. Oncol. 40 132-42.

Nakagawa K, Haga A, Shiraishi K, Yamashita H, Igaki H, Terahara A, Ohtomo K, Saegusa S, Shiraki T and Oritate T 2009 First clinical cone-beam CT imaging during volumetric modulated arc therapy Radiotherapy and Oncol. $90422-423$.

Ohnesorge B, Flohr T, Schwarz K, Heiken JP and Bae K T 2000 Efficient correction for CT image artifacts caused by objects extending outside the scan field of view Med. Phys. 27(1) 39-46.

Otto K 2008 Volumetric Modulated Arc Therapy: IMRT in a single gantry arc Med. Phys. 35 310-317.

Palma D, Vollans E, James K, Nakano S, Moiseenko V; Shaffer R, McKenzie M, Morris J and Otto K 2008 Volumetric modulated arc therapy for delivery of prostate radiotherapy: comparison with intensity-modulated radiotherapy and threedimensional conformal radiotherapy. Int. J. of radiation oncology, biology, physics 72(4) 996-1001.

Press W H, Teukolsky S A, Vetterling W T and Flannery B P 2002 B10: Minimization or Maximization of Functions Numerical recipes in Fortran 90 2nd Edition (Cambridge University Press) (New York, USA) pp.1201-1204.

Ramachandran G N and Lakshminarayanan A V 1971 Three dimensional reconstruction from radiographs and electron micrographs: applications of convolutions instead of Fourier transforms Proc. Natl. Acad. Sci. USA 68 2236-2240.

Snyder D L, O'Sullivan J A, Murphy R J, Politte D G, Whiting B R and Williamson J F 2006 Image reconstruction for transmission tomography when projection data are incomplete Phys. Med. Biol. 51(21) 5603-19.

Phys Med Biol. 2006 Nov 7;51(21):5603-19. Epub 2006 Oct 16.

Verbakel W F A R, Senan S, Cuijpers J P, Slotman B J, Lagerwaard F J 2009 Rapid delivery of stereotactic radiotherapy for peripheral lung tumors using volumetric intensitymodulated arcs Radiotherapy and Oncol. 93122-124.

Webb S 2010 Does the option to rotate the Elekta Beam Modulator MLC during VMAT IMRT delivery confer advantage?--a study of 'parked gaps' Phys. Med. Biol. 55(11) N303-19.

Webb S and McQuaid D 2009 Some considerations concerning volume-modulated arc therapy: a stepping stone towards a general theory Phys. Med. Biol. 54(14) 4345-60.

Yi B, Han-Oh Y, Lerma F, Berman B and Yu C MO-FF-A1-01: Fluoroscopic Verification of Intensity-Modulated Rotational Arc Therapy Delivery Med. Phys. 362708.

Yu C X 1995 Intensity-modulated arc therapy with dynamic multileaf collimation: An alternative to tomotherapy Phys. Med. Biol. 40 1435-1449. 
CT reconstruction from portal images acquired during VMAT

Zhou J, Zhang P, Turian J and Chu J 2009 SU-FF-J-81: A Feasibility Study for Real-Time Tomosynthesis-Guided Rapid Arc Therapy Med. Phys. 362494. 